\title{
Accelerating Apache Farms Through Ad-HOC Distributed Scalable Object Repository*
}

\author{
M. Aldinucci ${ }^{1}$ and M. Torquati ${ }^{2}$ \\ 1 Inst. of Information Science and Technologies - CNR, Via Moruzzi 1, Pisa, Italy \\ 2 Dept. of Computer Science - University of Pisa - Viale Buonarroti 2, Pisa, Italy
}

\begin{abstract}
We present HOC: a fast, scalable object repository providing programmers with a general storage module. HOC may be used to implement DSMs as well as distributed cache subsystems. HOC is composed of a set of hot-pluggable cooperating processes that may sustain a close to optimal network traffic rate. We designed an Hoc-based Web cache that extends the Apache Web server and remarkably improves Apache farms performances with no modification to the Apache core code.
\end{abstract}

Keywords: Web caching, cooperative caching, Apache, Web server, DSM.

\section{Introduction}

The demand for performance, propelled by both challenging scientific and industrial problems, has been steadily increasing in past decades. In addition, broadband networks growing availability has boosted Web traffic and therefore the demand for high-performance Web servers. Distributed memory Beowulf clusters are gaining more and more interest as low cost parallel architectures meeting such performance demand. This is especially true for industrial applications that require a very aggressive development and deployment time for both hardware solutions and applications, e.g. software reuse, integration and interoperability of parallel applications with the already developed standard tools.

We present HOC (Herd of Object Caches), a distributed object repository specifically thought for Beowulf class clusters. HOC provides applications with a distributed storage manager that virtualize processing elements (PEs) primary memories into an unique common memory. As we shall see, HOC is not yet another Distributed Shared Memory (DSM), it rather implements a more basic facility. It can be used as a DSM building block as well as for other purposes. Indeed, in this paper we present the design of a cooperative cache built on top of HOC for farms of the Apache Web server [1]. HOC provides Apache farms with a hot-pluggable, scalable cache that considerably improves Apache farms

* This work has been supported by the SAIB Project, funded by MIUR and leaded by SEMA S.p.A. by Athos Origin Group, on High-performance infrastructures for financial applications, and the Italian MIUR FIRB Grid.it project No. RBNE01KNFP. 
a)

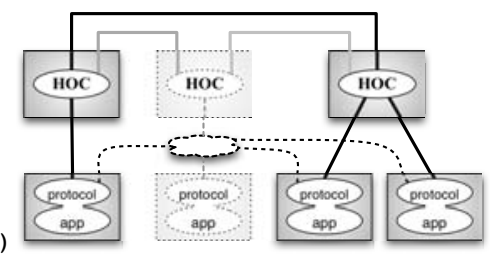

b)

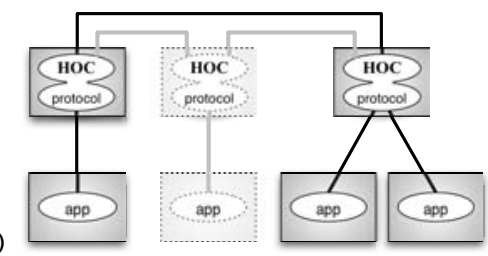

Fig. 1. Typical architectural schemes of applications based on HOC. a) With data access protocol within the application. b) Extending HOC external operations.

performance with no modification to the standard Apache 2.x core code, thus meeting key industrial requirements.

In Sec. 2 we present HOC design principles, HOC implementation and its raw performance. In Sec. 3 we discuss Web server test-bed peculiarities and HOC+Apache architecture. Experiments on such architecture are discussed in Sec. 4. The paper is completed by some conclusion.

\section{HOC Design Principles}

The HOC underlying design principle consists in clearly decoupling the management of computation and storage in distributed applications. The development of a parallel/distributed application is often legitimated by the need of processing large bunches of data. Therefore data storages are required to be fast, dynamically scalable and enough reliable to survive to some hardware/software failures. Decoupling helps in providing a broad class of parallel applications with these features while achieving very good performances.

HOC, like a DSM, virtualizes PEs primary memories in a common distributed repository. As a matter of fact, it realizes an additional layer of memory hierarchy that lays upon O.S. virtual memory, but that should be used instead of - or together with - disk-based virtual memory. The HOC implements an external storage facility, i.e. a repository for arbitrary length, contiguous segments of data (namely objects). Objects are identified by a configurable length key and have a home node, i.e. the reference node for the object (that is currently statically assigned to objects). It enables applications to fully utilize the underlying strength of a cluster, such as fast network communications and huge aggregated memory space. Current and near future network technologies clearly indicates that such a net-based virtual memory may perform much better than disk-based ones. Literature reports very good results both for general DSMs [2,3] and for specific Web applications [4-6]. A large and fast data repository may be used as a cache facility to improve performance of I/O bound applications, and as a primary storage facility for CPU bound applications running out-of-core on a single PE memory.

HOC, unlike some DSMs, is quite robust and simple. It does not natively implement any consistency mechanism for data copies and does not force already developed applications to be rewritten. As shown in Fig. 1, the Hoc-based 

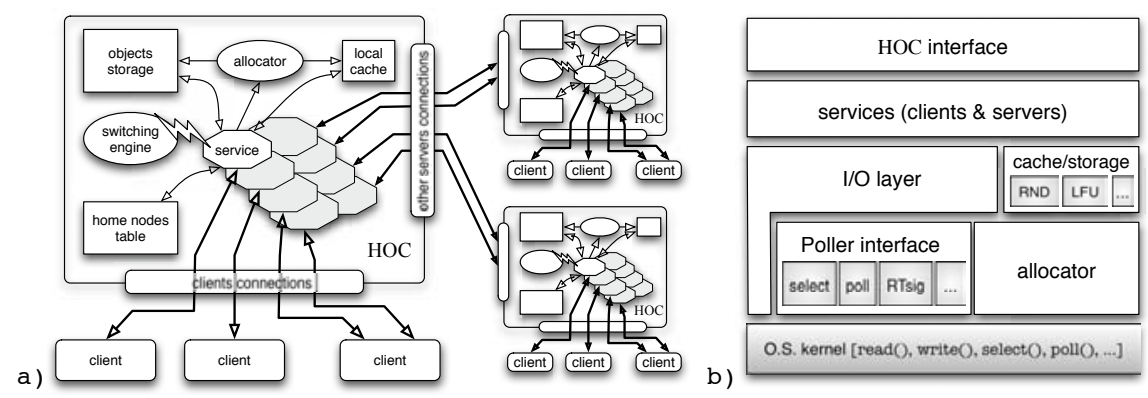

Fig. 2. HOC architecture. a) Functional view. b) Implementation layers.

architecture distinguishes the raw data access layer from the protocol each application requires to access data. In many cases, for example in the context of Web caching, a sophisticated data consistency mechanism is not really needed and may introduce unnecessary overheads. However, HOC may be enriched with locking and consistency mechanisms at the protocol level (as shown in Fig. 1 a).

\subsection{HOC Implementation}

A HOC server is implemented as a $\mathrm{C}++$ single thread process; it relies on nonblocking I/O to manage concurrent TCP connections $[7,8]$. The HOC core consists of an executor of a dynamic set of finite state machines, namely services, each of them realized as a $\mathrm{C}++$ object. Each service reacts to socket-related events raised by O.S. kernel (i.e. connections become writable/readable, new connection arrivals, connection closures, etc.). In the case one service must wait on an event, it consolidates its state and yelds the control to another one. The HOC core never blocks on I/O network operations: neither on read()/write() system calls nor on HOC protocol primitives like remote node memory accesses. The event triggering layer is derived from the Poller interface [9], and may be configured to use several POSIX connections multiplexing mechanisms, such as: select, poll, and Real-Time signals.

HOC architecture is sketched in Fig. 2. A HOC may serve many clients, each of them exploiting many connections. A server may cooperate with other HOCs through a configurable number of connections. Connections both with clients and other servers may be established or detached at any time during HOC lifespan.

A connection is exclusively managed by a service. Services rely on an allocator in order to store objects into both a object storage and a write-back cache facilities. They may be both managed as a cache of a configurable but fixed amount of objects and used to store server home objects and to cache remote home objects respectively. As shown in Fig. 2 b) each of them may be managed by using a replacing policy chosen at configuration time. Currently HOC comes with RND (random) and LFU (Least Frequently Used) policies, however it is designed to be easily extended with other policies. HOC offers three basic objectrelated services (external operations): get, put, and remove. Assuming to have a set of nodes each of them running an $\mathrm{HOC}$, and $\mathrm{HOC}_{i}$ receive: 
$-\operatorname{get}(x)$. If home $(x)=i$, a local object storage_get $(x)$ is issued. If otherwise home $(x)=j, j \neq i$ a local cache_get( $x)$ and, in case of miss, a remote_get $(x, j)$ is issued. In the latter case the object $(x)$ is stored in the $i$ local cache. Anyway, depending on the get results, either the object $(x)$ or a miss message is sent to the client.

$-\operatorname{put}(x)$. If home $(x)=i, \operatorname{object}(x)$ is put in the node $i$ storage. Otherwise $\operatorname{object}(x)$ is stored in the node $i$ local cache. A capacity miss can occur in both cases. In the former case the victim object is simply deleted ${ }^{1}$, in the latter the write-back reconciling protocol is started.

- remove $(x)$. The object $(x)$ is purged from both object storages and caches of all nodes.

Overall, HOC implements a Multiple Reader Single Writer protocol. Observe that HOC does not natively implement any memory consistency and does not provide any locking facility. These features are supposed to be realized at the protocol level as shown in Fig. 1 a).

\subsection{HOC Performances}

HOC performances has been extensively tested on several homogeneous clusters. We report here tests performed on a 21 PEs RLX Blade; each PE runs Linux (2.4.18 kernel) and is equipped with an Intel P3@800MHz, 1GB RAM, a 4200rpm disk and three 100Mbit/s switched Eth devices. As shown in Fig. 3, HOC scales very well up to the maximum number of PEs available in our testing environment (10 HOCs and 10 clients). In the tests two different Ethernets are used for client-server and server-server connections. As shown in Fig. 3 b), HOC can deliver an aggregate throughput that is quite close to the $100 \mathrm{Mbit} / \mathrm{s}$ per PE asymptotic network bandwidth. We experienced a little impact of local cache size on performances. Cache mainly acts as a network buffer.

Other tests performed on a Linux (2.4.22 kernel) cluster of 8 PEs P4@2GHz, $512 \mathrm{MB}$ RAM, connected through a $1 \mathrm{Gbit} / \mathrm{s}$ Eth have confirmed for a single HOC and many clients an aggregate throughput of $\sim 91 \mathrm{MB} / \mathrm{s}(\sim 96 \%$ of the measured $95 \mathrm{MB} / \mathrm{s}$ maximum bandwidth) with 2048 concurrent stable connections for a test of 50,000 requests of $1 \mathrm{MB}$ objects. We also experienced more than 20,000 replies/s with 3,072 concurrent stable connections for a test of 200,000 requests of $512 \mathrm{~B}$ objects. Actually, HOC has sustained a throughput close to the network bandwidth in any tested case.

\section{$3 \quad$ Web Caching Test-Bed}

Web caches have became the standard method to ensure high-quality throughput for Web access. Web caching can be adopted at different levels: 1) Web browser storing Web objects in its local memory or disk. 2) proxy server sitting somewhere between the clients and the Web server (typically at ISP level) serving a

\footnotetext{
${ }^{1}$ If configured as a cache, otherwise a storage full message is sent to the client.
} 

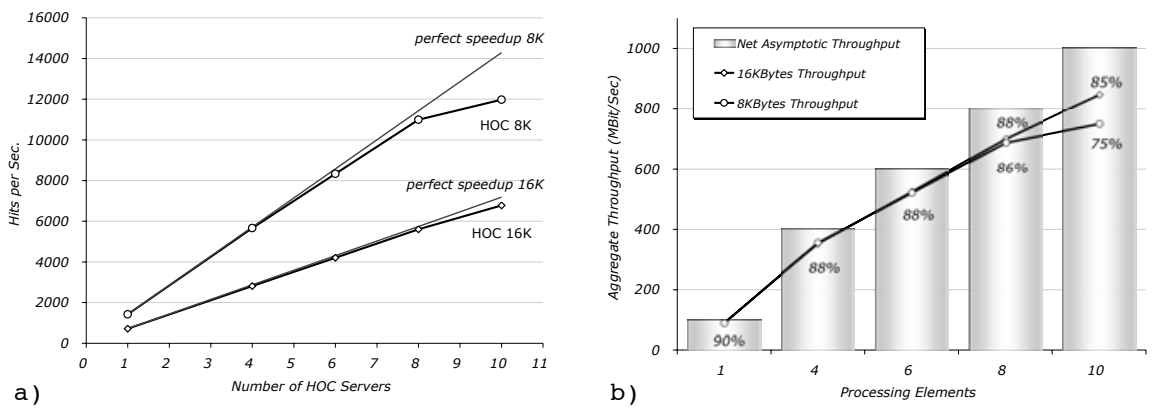

Fig. 3. a) HOC scalability of served requests. b) Aggregate throughput. Experiments are referred to 200,000 get operations w.r.t. a data set of 40,000 objects ( $8 \mathrm{~K}$ and $16 \mathrm{~K}$ average sizes) having cyclically distributed homes among HOCs. Each HOC serves a partition of requests referred to keys which are uniformly distributed and randomly chosen in the whole data set. Each HOC have a local cache of 1,000 objects.

large community of clients. 3) server accelerator (reverse proxy) sitting in front of one or more Web servers [10,11].

Reverse proxies have been demonstrated to be the best solution among them. Differently from 1 and 2, they are under the site manager control and typically enable dynamic pages management through an API which allows application programs to explicitly add, delete, and update cached data $[12,13]$. However, a reverse proxy may introduce unnecessary latencies due to the additional parsing and filtering of requests, including those leading to not cacheable replies. To mitigate these problems we adopt a different solution: we place a cache on back of the Web server in order to improve server performances. The cache cope with cacheable replies only and does not make any additional parsing on them. As we shall see in the next section, we implemented the solution by using a HOC-based architecture. Our approach is similar to others appeared in literature $[14,4,6,5]$. Differently from other approaches, we did not designed another Web server, we are rather proposing a decoupled architecture which is composed of the standard Apache Web server and a HOC-based storage subsystem. Apache Web server is unmodified in core functionalities, we just modified the allocation policy of one optional Apache module. Therefore the architecture may benefit from Apache popularity, correctness and people expertise.

In a cache implemented on a single node (single or even multi-processor), the throughput is limited by the network interface. With cluster based Web cache the throughput can be increased simply adding more nodes. Since many HOCs may cooperate with each other, the cache throughput is not limited by the network interface, but by the aggregate cluster throughput.

\subsection{HOC+Apache Architecture}

The HOC+Apache architecture is compliant to Fig. 1 a). In this case the app is the Apache Web server, the protocol is a modified version of mod_mem_cache Apache 
Table 1. Experimental environment summary. a) Raw data set characteristics. b) Access log characteristics. c) Apache Web server configuration.

a) Raw data set

\begin{tabular}{ll}
\hline Total size & $4 \mathrm{~GB}$ \\
N. of files & 100,000 \\
N. of requests & 250,000 \\
N. of files $<256 \mathrm{~KB}$ & $96209(2 \mathrm{~GB})$ \\
Static pages & $100 \%$ \\
\hline
\end{tabular}

b) Accesses log

\begin{tabular}{ll}
\hline Data transfered & $\sim 9 \mathrm{~GB}$ \\
$\mathrm{~N}$. of distinct files requests & $74226(2.8 \mathrm{~GB})$ \\
Avg. file size & $36.8 \mathrm{~KB}$ \\
$\mathrm{~N}$. of distinct files $<256 \mathrm{~KB}$ & $71449(1.5 \mathrm{~GB})$ \\
Avg. size of distinct files $<256 \mathrm{~KB}$ & $8 \mathrm{~KB}$ \\
\hline
\end{tabular}

c) Apache 2.0.47 MPM worker configuration (hybrid multi-threaded multi-process) [1]

\begin{tabular}{llllllll}
\hline StartServers & 4 & MaxClients & 512 & ThreadPerChild & 64 & Log level & Notice \\
ServerLimit & 8 & MinSpareThreads & 32 & MaxRequestsPerChild & 0 & Access log & None \\
\hline
\end{tabular}

module [1] (compliant to RFC2616), and dashed lines are not present. In particular, mod_mem_cache has been modified by only substituting local memory allocation, read and write with HOC primitives. The work-flow of the mod_mem_cache modified to inter-operate with HOC is sketched in Fig. 4 a). No other functionalities have been modified. Both original and modified caches are able to cache static and dynamic pages. Note that the original version of mod_mem_cache implements a per process cache, thus different Apache processes never share the cache even if they are running on the same PE.

We used as objects key the MD5 digest of the mod_mem_cache native key. In order to enforce correctness and consistency we include in the object to be stored into HOC original HTTP request and reply headers. Protocol does not need any additional consistency mechanism but the ones ensured by mod_mem_cache.

Observe that $\mathrm{HOC}+$ Apache architecture is designed to improve Apache performance whether the performance bottleneck is memory size, typically in the case the data set does not fit the main memory. In all other cases (e.g. in-core data sets), the HOC+Apache architecture does not introduce performance penalties w.r.t. the stand-alone Apache equipped with the native cache.

\section{Web Caching Experiments}

We measured the performance of Apaches+HOC architecture on the RLX Blade described in Sec. 2.2. The main characteristics of the data set and accesses log are summarized in Table $1 \mathrm{a}$ ) and b). The data set is generated according to [15] by using a Zipf-like request distribution with $\alpha=0.7$. 90\% of files have small size (min. $2 \mathrm{~KB}$, max. 100KB, avg. $\sim 13 \mathrm{~KB}$ ) and $10 \%$ of files have medium-large size (min. 101KB, max. 1MB, avg. $\sim 280 \mathrm{~KB}$ ). In all tests we used the Apache 2.0.47 Web server in the MPM Worker configuration shown in Table $1 \mathrm{c}$ ). Files greater than $256 \mathrm{~KB}$ are not cached. HTTP requests are issued by means of the httperf program [16] configured to count replies only within 1 second timeout. Each httperf is directly connected to an Apache with no switch/balancer in the middle. The whole site is replicated on all PEs running Apache.

In Fig. 4 b) we compare Apache against Apache+HOC performances. The test takes in to account three basic classes of configurations: 


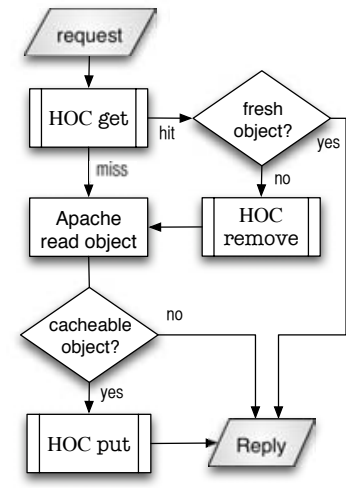

a)

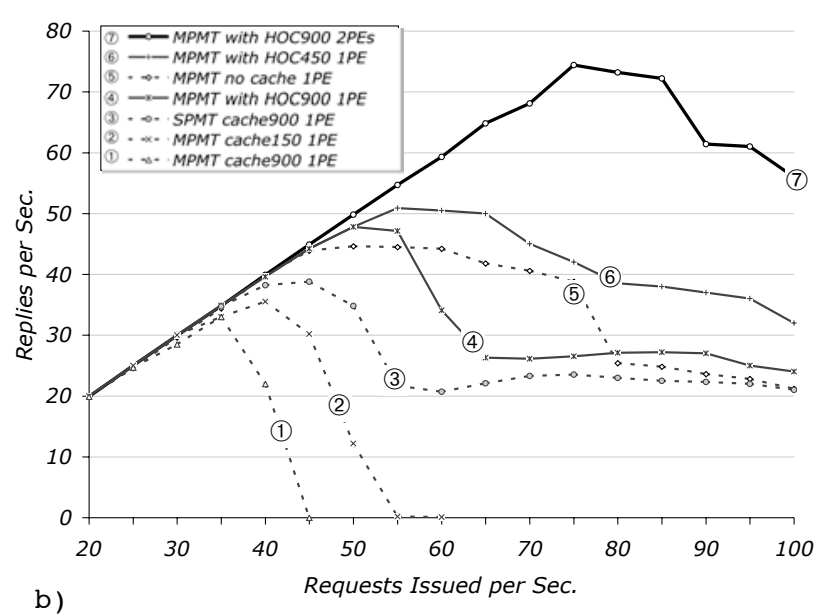

b)

Fig. 4. a) High-level work-flow of mod_mem_cache changes needed to inter-operate with HOC. b) Replies rate for stand-alone Apache in the Multi-Process Multi-Threaded (MPMT) and Single-Process Multi-Threaded (SPMT) configurations and HOC+Apache architecture. Apache is tested with $900 \mathrm{MB}$ and $150 \mathrm{MB}$ native cache per process and without cache. HOC+Apache is tested with $450 \mathrm{MB}$ and $900 \mathrm{MB}$ devoted to HOC (1PE). HOC+Apache is also tested with Apache and HOC (900MB) on different PEs (2PEs).

i) a stand-alone multi-process multi-threaded Apache with no cache (5), with the mod_mem_cache (Apache native cache) exploiting both $900 \mathrm{MB}$ (1) and $150 \mathrm{MB}$ cache (2) per process. A stand-alone Apache configured as one server process exploiting 512 threads sharing the same $900 \mathrm{MB}$ cache (3);

ii) an Apache+HOC running on the same PE, HOC exploiting both 450MB (6) and $900 \mathrm{MB}$ (4) of total memory accessed by all Apache processes;

iii) an Apache+HOC running on different PEs, HOC exploiting 900MB of total memory accessed by all Apache processes ( ( $)$.

The three cache sizes $150 \mathrm{MB}, 450 \mathrm{MB}, 900 \mathrm{MB}$ have an hit rate of $29 \%, 45 \%$, $60 \%$ respectively when tested on a single Apache native cache. As clear from Fig. 4 b), in all cases the HOC+Apache architecture overwhelms the stand-alone Apache with or without the native cache, including the case the native cache is shared among all running threads.

i) We have observed that the Apache with the original cache lose its stability when the requests rate grows. This is mostly due to the lack of a common cache storage for all processes on the same $\mathrm{PE}$, which leads to the replication of the same objects in several caches, and therefore to the harmful memory usage (1), (2). This rapidly leads the O.S. to the swap border resulting in a huge increase of reply latency. Indeed, Apache configured as SPMT thus exploiting a single shared native cache (3) perform better than MPMT configurations. Quite surprisingly the Apache with no cache performs even better (5)). In reality this behavior is due to the File System buffer that acts as a shared cache for all Apache 


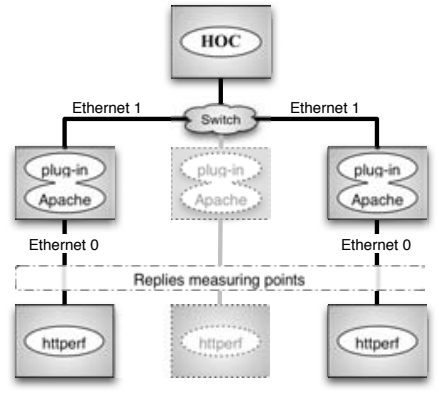

a)

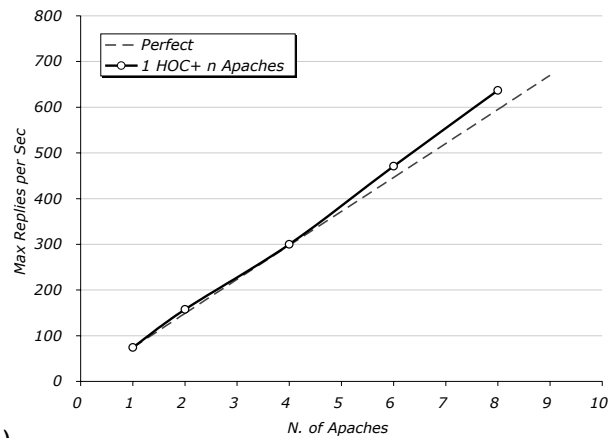

b)

Fig. 5. One HOC supporting many Apaches. a) Experiment schema. b) Replies per second speedup. HOC is configured with $900 \mathrm{MB}$ of objects storage. Dataset and Apache configuration are summarized in Table 1.

processes coping with object replication problem. Actually, the FS buffer rise up to $\sim 700 \mathrm{MB}$ during test (5). In this case the performance also depends on site organization on disks. In general FS cache is unsuitable for Web objects since requests do not exploit spatial and temporal locality w.r.t. disk-blocks [15]. Moreover, FS cache is totally useless for dynamic Web pages, for which we experienced the effectiveness of the Apache native cache module [12].

ii) The Apache+HOC architecture performs better than stand alone Apache, even if Apache and HOC are mapped on the same PE (4), (6)). Apache processes share a common memory through HOC. Since the accessed set of files does not fit in memory, performance may be influenced by replacing policies. Here, two different caching mechanism and three policies are active at the same time: 1) the FS buffer and HOC allocates memory in different bulks from the same physical memory; 2) the FS buffer replacing policy is active on the first bulk, the Apache GreadyDual-Size and the HOC LFU replacing policies are active in cascade on the second bulk. The knotty scenario prevents a fine analysis of system bottlenecks. Indeed, the decoupling approach we followed sought to simplify the design in order to make effective the system tuning.

iii) As a matter of fact, the 2PEs figures (7) confirm that mapping Apache and HOC on different PEs significantly improves performances. The same tests have been performed using as HOC replacing policy the random function instead of LFU. The performance degradation is just 8.7\% (from 74.4 to 67 avg. replies). The replacing policy have a little impact on performances in this case, whereas the decoupling of cache management from the server activity significantly improves architecture stability and performance. In fact decoupling distributes the memory pressure due to the Apache server and its cache on different PEs.

Figure 5 highlights that HOC may support many Apaches with a very good, over linear scalability due to the partition of requests among several Apaches (which induces a lower disk load). Note that HOC+8Apaches sustain an aggregate of 637 hits/s with an average file size of $8 \mathrm{~KB}$ on the Apache-HOC link (see Table $1 \mathrm{~b})$. In this case HOC works at $\sim 45 \%$ of maximum reply rate since HOC reaches 1430 hit/s with $\mathrm{N}=1$ and the same message payload, see Fig. 1. 


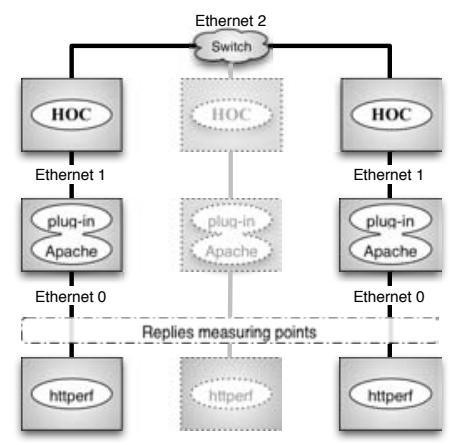

a)

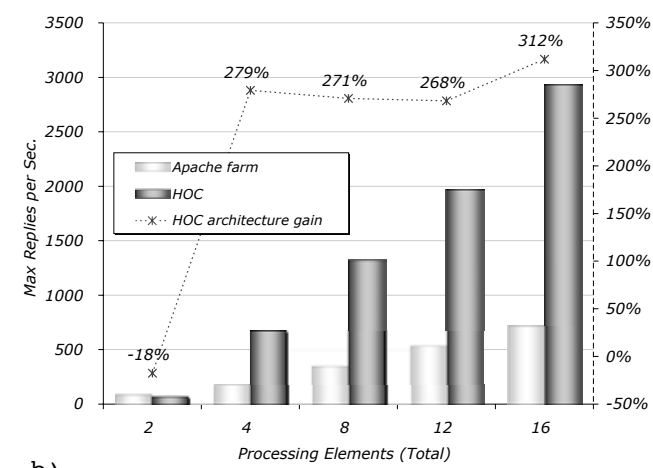

b)

Fig. 6. Many cooperating Hocs experiment. a) Experiment schema. b) Dark bars shows the replies/s speedup w.r.t. the total amount of used PEs ( $n$-HOCs and $n$-Apaches). Light bars shows the same measure for a reference architecture ( $2 n$-Apaches on the fastest tested configuration, see Fig. 4). Dashed line sketch the HOC-based architecture gain. The HOC local cache is fixed to 5,000 objects.

Figure 6 shows the scalability of $n$ Apache $+n$ HOC. Also in this case we experienced a very good scalability up to our testing environment limit reaching a $312 \%$ gain w.r.t. an Apache farm (8HoCs+8Apaches vs 16 Apaches). If compared with Apache with the original cache module the gain reaches the $406 \%$. Observe that the big gap occurs from $1 \mathrm{HOC}+1$ Apache and $2 \mathrm{HOC}+2 \mathrm{Apaches}$, where the whole set of all cacheable objects begins to fit in the aggregate memory.

\section{Conclusion}

We introduced HOC, a fast and scalable "storage component" for homogeneous cluster architectures. HOC implements a distributed storage service relying on state-of-the-art server technologies. As described in Sec. 2.2, these enable HOC to cope with a large number of concurrent connections and to sustain a very high throughput in both single and parallel configurations. We developed and tested a HOC-based Apache plug-in module which greatly improves Apache Web server farms performances. To the best of our knowledge, no other works target the problem with no modification to a preexisting centralized Web server.

We are currently improving HOC in two directions. First, by introducing multithreading to make it scalable also on SMP boxes. Second, by integrating HOC within the ASSIST parallel programming environment [17]. Overall, we envision a complex application made up of decoupled components, each delivering a very specific service. Actually, HOC provides the programmer with a data sharing service [18]. In this scenario, the application or the programming environment run-time support is supposed to provide the correct protocol to consistently use HOC external operations. At this end we are developing a protocol which offers standard hooks to implement several DSM consistency models [3, 2]. 


\section{Acknowledgments}

We wish to thank M. Danelutto and M. Vanneschi for many fruitful discussions, and A. Petrocelli who has contributed in experimenting HoC.

\section{References}

1. Apache Software Foundation. Apache Web Server, 2003. (http://httpd.apache.org).

2. M. Aldinucci. eskimo: experimenting with skeletons in the shared address model. Parallel Processing Letters, 13(3):449-460, September 2003.

3. G. Antoniu and L. Bougé. DSM-PM2: A portable implementation platform for multithreaded DSM consistency protocols. In Proc. of the 6th Intl. HIPS Workshop, number 2026 in LNCS. Springer, April 2001.

4. G. Chen, C. L. Wang, and F. C. M. Lau. A scalable cluster-based web server with cooperative caching support. Computation and Currency: Practice and Experience, 15(7-8), 2003.

5. F. M. Cuenca-Acuna and T. D. Nguyen. Cooperative caching middleware for cluster-based servers. In Proc. of the 10th IEEE HPDC, August 2001.

6. E. Cecchet. Whoops! : A clustered web cache for dsm systems using memory mapped networks. In Proc. of the 22nd IEEE ICDCSW Conference, July 2002.

7. D. Kegel. The C10K problem, 2003. (http://www.kegel.com/c10k.html).

8. A. Chandra and D. Mosberger. Scalability of Linux event-dispatch mechanisms. Technical Report HPL-2000-174, HP Labs., Palo Alto, USA, December 2000.

9. D. Kegel. Poller Interface, 2003. (http://www.kegel.com/poller/).

10. J. Wang. A survey of Web caching schemes for the Internet. ACM Computer Communication Review, 29(5), 1999.

11. M. Rabinovich and O. Spatsheck. Web Caching and Replication. A. Wesley, 2001.

12. A. Iyengar and J. Challenger. Improving Web server performance by caching dynamic data. In Proc. of the USENIX Symposium on Internet Technologies and Systems Proceedings, Berkeley, CA, USA, Dec. 1997.

13. B. Hines. Livin' on the Edge: Caching Dynamic Web Content with IBM WebSphere Application Server and WebSphere Edge Server. IBM, September 2001. (http://www-106.ibm.com/developerworks/websphere/techjournal/ 0109_hines/hines.html).

14. Whizz Technology. EC-Cache Engine, 2003. (http://www. whizztech.com/ec_cache.html).

15. L. Brelau, P. Cao, L. Fan, G. Phillips, and S. Shenker. Web caching and Zipf-like distributions: Evidence and implications. In Proc. of the Infocom Conference, 1999.

16. D. Mosberger and T. Jin. httperf - a tool for measuring Web server performances. In Proc. on Internet Server Performance (WISP), Madison, USA, June 1998.

17. M. Vanneschi. The programming model of ASSIST, an environment for parallel and distributed portable applications. Parallel Computing, 28(12), Dec. 2002.

18. G. Antoniu, L. Bougé, and M. Jan. Juxmem: An adaptive supportive platform for data sharing on the Grid. In Proc. of IEEE/ACM Workshop on Adaptive Grid Middleware (in conjunction with PACT 2003), New Orleans, USA, September 2003. 